\title{
Análisis de la demanda del servicio eléctrico de los hogares de la Parroquia Jambelí
}

\author{
Lady Andrea León Serrano
}

Universidad Técnica de Machala, Ecuador

Autor para correspondencia: 1lady@utmachala.edu.ec

ORCID: http://orcid.org/0000-0001-5472-140X

Fecha de recepción: 10 de enero de 2020

Fecha de aceptación: 05 de abril de 2020

\section{Resumen}

Los cambios de comportamiento en la adquisición de servicios básicos han repercutido en los niveles de consumo de cada localidad de la ciudad de Machala, aun cuando las características de los habitantes son diferentes por la calidad de vida, han incidido en el factor social, familiar y renta. Por tal motivo, el objetivo de la investigación fue determinar la demanda de servicio eléctrico de los hogares de la Parroquia Jambelí, ciudad de Machala, para cumplir con el objetivo fue necesario establecer la localidad y tipo de instrumento de aplicación que influyeron en la variación del consumo de energía eléctrica. La metodología fue de tipo exploratoria, descriptiva y de alcance correlacional, se aplicó el instrumento de investigación correspondiente a una ficha tipo encuesta dirigida a las familias del sector, los datos fueron procesados mediante modelación econométrica. Los principales resultados demostraron que el modelo aplicado es acertado y las variantes tienen incidencia en la demanda de dicho servicio básico. Las conclusiones generales establecieron que el nivel de ingresos, la edad, número de integrantes por hogar, número de artefactos eléctricos, temporada y tipo de vivienda (edificio) fueron factores claves para el aumento del consumo de electricidad, afectando la capacidad económica de los hogares, así como la necesidad de políticas públicas para mantener un consumo responsable.

Palabras clave: Demanda, Consumo, Servicio Eléctrico, Ingreso familiar, Hogares.

\section{Abstract:}

The behavioral changes in the acquisition of basic services have had an impact on the consumption levels of each locality in the city of Machala, even though the characteristics of the inhabitants are different due to the quality of life, have influenced the social, family and rent. For this reason, the objective of the research is to determine the demand for electrical service in the homes of the Jambelí Parish, city of Machala. To meet the objective, it was necessary to establish the location and type of application instrument that influenced the variation of the electric power consumption. The methodology was exploratory, descriptive and correlational in scope, the corresponding research instrument was applied to a survey type sheet addressed to families in the sector, the data were processed using econometric modeling. The main results demonstrated that the applied model is successful and the variants have an impact on the demand for said basic service. The general conclusions established that the level of income, age, number of members per household, number of electrical appliances, season and type of dwelling (building) were key factors for the increase in electricity consumption, affecting the economic capacity of households. , as well as the need for public policies to maintain responsible consumption. 
Keywords: Demand, Consumption, Electric Service, Family Income, Homes.

\section{Introducción}

En la actualidad, la vida moderna ha presentado cambios sustanciales en los comportamientos de consumo de servicios básicos, las redes de conexiones a nivel global basadas en tecnologías están impulsando mayor fuente de ingresos en los sistemas económicos, cada vez existe un mayor consumo del suministro de energía eléctrica en los sectores productivos por la vital necesidad de producir bienes y servicios. (Álvarez, 2016) expone, el proceso de liberalización de la industria eléctrica, al igual que otros sectores tradicionalmente implica que los precios sean competitivos, así como el factor humano necesita de esta fuente indispensable para llevar sus actividades particulares y llegar a obtener un confort mínimo.

La energía eléctrica es un servicio básico indispensable para la vida diaria, para las familias, los mecanismos de subsidios planteados por distintas economías están adaptadas para los diferentes tipos de usuarios que a su vez se rigen por la política energética estatal. Así la producción y consumo de energía en nuestros días se relacionan con el desarrollo sostenible como un esquema socioeconómico que involucra y afecta a los habitantes del planeta.

El crecimiento poblacional urbano en las principales ciudades de las naciones, es un desafío para la formulación de políticas públicas e inversionistas privados, hoy en día la mayoría de la población mundial se concentra en las grandes ciudades (Pavón \& Barzola, 2015). América Latina es una de las franjas de mayor consumo, para el caso Ecuador, los factores que inciden en este servicio es la renta percibida por los hogares. El propósito del tema de investigación es determinar la demanda de servicio eléctrico de los hogares de la Parroquia Jambelí, ciudad de Machala; con esa intención para cumplir el objetivo fue necesario aplicar una encuesta al público objetivo, obteniendo resultados referentes a los aspectos que influyen en el aumento tarifaria del servicio básico.

De acuerdo con datos publicados en el Plan Maestro de Electrificación, el Ecuador pasó de un promedio del 5,5\% en el incremento anual de la demanda de electricidad registrado entre el 2000 y 2012, a tasas de crecimiento que llegaron hasta el 21,3\% anual en el 2017, provocado por el aumento de la población y el mejoramiento de su estilo de vida que se ve reflejado en un mayor consumo (OLADE, 2019), estos planteamientos justifican el estudio para ser aplicados en sectores micro de los cantones principales, para el presente caso refiere a la localidad de estudio.

Los hábitos de consumo de electricidad de la ciudad de Machala en diversos sectores residencial, comercial, industrial y acceso público, están en concordancia con la capacidad de respuesta de los centros de acopio de abastecimiento del estado. El problema surge por los cambios en los hábitos de consumo, regeneración urbana, calidad de vida de los habitantes, clima, que permiten comprender como este rubro puede disminuir la liquidez

Esta obra se comparte bajo la licencia Creative Common Atribución-No Comercial 4.0 International (CC BY-NC 4.0) 
ante un consumo no controlado por las economías domésticas.

El alcance del estudio refiere a los hogares que habitan en la Parroquia Jambelí de la ciudad de Machala y su comportamiento frente a la demanda del servicio eléctrico, la importancia se conserva en conocer las variables que influyen en la investigación y las necesarias políticas públicas para el control de los servicios básicos de la localidad, que permita ahorrar y llegar a más sectores vulnerables.

En base a lo anterior, la metodología es de tipo exploratoria y descriptiva, de alcance correlacional, los resultados obtenidos luego de la aplicación del instrumento de investigación fueron tabulados mediante modelación econométrica destacando que la elasticidad ingreso de corto plazo de la demanda eléctrica (considerando al hogar como unidad de análisis) es de 0,48; la elasticidad integrantes del hogar es 0,27 ; la elasticidad artefactos eléctricos llega a 0,56; la elasticidad edad es 0,26; la elasticidad por temporada es 0,53 y elasticidad edificio es 0,24 , es decir, cada una de las variantes planteadas influyen significativamente en el aumento de consumo de energía eléctrica, destacando que este rubro afecta el ahorro familiar.

El tema es de interés académico y de la sociedad para entender el consumo familiar ante escasos recursos y cambios sustanciales de la población que influyen a mejorar la calidad de los servicios ante una demanda eléctrica que siempre está insatisfecha.

\section{Fundamentos teóricos}

El consumo de energía eléctrica constituye una de las prioridades de agenda económica, política, y social. Los países con mayor pobreza muestran bajos niveles de consumo por la escasa disponibilidad, mientras los países desarrollados con mejores niveles de ingresos presentan grandes cantidades de consumo, en el caso de las economías en vías de desarrollo experimentan drásticos aumentos poblacionales ocasionando el aumento de la demanda del servicio eléctrico, consecuentemente, las empresas permiten garantizar el equilibrio entre el desarrollo de un mercado y la atención del rubro. (Morales \& Alvarado, 2014)

El acto de adquirir o gastar un bien o servicio se lo llama consumo, esto permite a las economías mantenerse en constante actividad por la diversidad de productos, además se entiende como la etapa final de un proceso económico, los hábitos de consumo cada vez son más elevados, generando un mayor circulante para la economía local, pero afectando al ahorro y liquidez de los hogares (Carosio, 2008). Para Keynes el consumo dinamiza el entorno de una economía debido al estímulo de la demanda dando estabilidad al sistema productivo; Marx lo relacionaba con los factores dependientes del lugar que ocupen en la sociedad, sea capitalista u obrero, determinando las preferencias al momento de consumir (Caporaso \& Levine, 1992).

Así la demanda es la cantidad total de un bien o servicio que la gente desea adquirir en el mercado, tiene una amplia variedad de bienes y servicios para ser adquiridos a precios considerables por un consumidor o por el total de compradores en un lugar en específico, a fin

Esta obra se comparte bajo la licencia Creative Common Atribución-No Comercial 4.0 International (CC BY-NC 4.0) 
de poder satisfacer necesidades y deseos, es decir busca la manera más eficiente de asignar los recursos que son limitados (Mejía, 2016). El crecimiento económico de un país también es asumido por el aumento de consumo de energía eléctrica, varios países exportan o importan por la facilidad del transporte por medio de cables.

Estos cambios proporcionales entre una variable " $x$ " en relación de otra " $y$ ", refiere a la sensibilidad de la oferta y la cantidad demandada cuando existe diferencias en los precios, para esto presentamos la división de la demanda:

- Elástica, cuando la elasticidad de la demanda es mayor que 1, la variación de la cantidad demandada es porcentualmente superior a la del precio.

- Inelástica, cuando la elasticidad de la demanda es menor que 1, la variación de la cantidad demandada es porcentualmente inferior a la del precio

- Elasticidad unitaria, cuando la elasticidad de la demanda es 1, la variación de la cantidad demandada es porcentualmente igual a la del precio. (García, 2010)

Los diferentes tipos de elasticidades, son fundamentales para conocer la variación del consumo de un servicio básico en una economía, empresa o familias.

En el Ecuador, se proyecta que para el 2030, el consumo de electricidad experimente un incremento de 2.5\% anual (Castells, 2011). Según Decreto Ejecutivo No. 2713 del 7 de junio de 2002, establece la codificación del reglamento de tarifas eléctricas (ARCONEL, 2002), en tres categorías de tarifas:

- Residencial: Corresponde al servicio eléctrico destinado a uso doméstico de los consumidores dentro de la residencia de la unidad familiar de la carga conectada. Dentro de esta categoría se incluye a los consumidores de escasos recursos y bajos consumos que 24 Registro Oficial No. 598 de 17 de junio de 2002. Estudio sobre el Servicio de Energía Eléctrica en el Ecuador y su impacto en los consumidores tiene integrada a su vivienda una pequeña actividad comercial o artesanal.

- General: Pertenece al servicio eléctrico destinado a los consumidores que realizan actividades diferentes a la categoría residencial, comprende el comercio, la prestación de servicios públicos y privados, la industria. Por ejemplo: locales o establecimientos industriales, servicios de telecomunicaciones, tiendas, organismos internacionales, entidades del sector público, etc.)

- Alumbrado público: Se aplica a los consumos destinados al alumbrado de calles, avenidas, vías de circulación pública, iluminación de plazas, parques, monumentos, y a los sistemas de señalamiento luminoso para el control de tránsito.

Los hogares de la Provincia de el Oro son los que mensualmente pagan más por consumo de energía eléctrica en el país, considerando a los hogares con ingresos menores al sueldo básico, con un valor promedio de la tarifa que asciende a USD 21.88 superior a la media nacional que paga en torno a USD 16.97, así la ciudad de Machala tiene un consumo promedio de USD 18.74, menor que Guayaquil y Quito (ARCONEL, 2011).

Sin embargo, el consumo se agudiza a medida que los niveles de ingresos mejoran en las

Esta obra se comparte bajo la licencia Creative Common Atribución-No Comercial 4.0 International (CC BY-NC 4.0) 
diferentes ciudades del Ecuador, la investigación se establece con aplicación de encuestas en la Parroquia Jambelí, por ser un sector menos comercial y conformado por más hogares. Otro aspecto considerable es el clima de la región que supera en algunos casos los $23^{\circ}$ centígrados, provocando aumento en la adquisición de artefactos eléctricos, el incremento de miembros del hogar son factores claves del uso del servicio eléctrico.

\section{Materiales y métodos Método de investigación}

Para el desarrollo de la investigación, y determinar la demanda de servicio eléctrico en los hogares de la Parroquia Jambelí, ciudad de Machala, se ha procedido a desarrollar un test/ encuesta aplicado en el lugar de campo dirigido al público objetivo (hogares), consta de elementos cuantitativos y cualitativos que permitieron establecer las variables en el tema propuesto. De acuerdo (Del Canto \& Silva, 2013), el método cuantitativo se basa en la indagación a través de elementos cognitivos y en datos numéricos extraídos de la realidad, procesados estadísticamente para probar teorías. Además, es base para la resolución del planteamiento del tema, mediante los procesos de recolección de datos.

\section{Tipo de investigación}

El diseño de investigación es descriptivo por la referencia a características y perfiles de individuos o grupos que son sometidos a un análisis (Monje, 2011), en los resultados. El alcance del estudio es correlacional por la relación o grado de asociación que exista entre dos o más conceptos, categorías o variables en un contexto en particular (Hernández, Fernández, \& Baptista, 2010), es decir la vinculación de las variantes con respecto a la dependiente consumo de energía eléctrica de los hogares.

\section{Población y muestra}

Para el desarrollo del presente estudio se determinó la población, correspondiente 101 hogares de la Parroquia Jambelí que demandan el servicio de energía eléctrica. Se aplicó una encuesta a los jefes de hogar para el levantamiento de datos durante el primer semestre del año 2019, luego fueron tabulados mediante procedimiento econométrico determinando la relación con las variables planteadas.

\section{Técnica de Análisis de Regresión}

Según Gujarati \& Porter (2010), plantea que el análisis de regresión involucra el estudio de la relación entre dos variables cuantitativas. El modelo consistió en una regresión lineal múltiple con análisis Ancova, definido por (Cayuela, 2010) es posible que aparezcan tanto variables explicativas continuas como categóricas. La demanda del servicio eléctrico en función del ingreso, numero promedio de integrantes en el hogar, numero promedio de artefactos eléctricos en el hogar, temporada de consumo y tipo de vivienda.

Esta obra se comparte bajo la licencia Creative Common Atribución-No Comercial 4.0 International (CC BY-NC 4.0) 
Tabla. 1. Presentación y descripción de variables

\begin{tabular}{|c|c|c|c|c|c|}
\hline Clasificació & Nombre & Descripción & Tipo & Uni & Sign \\
\hline n de la Variable & de Variable & & & dades & o Esperado \\
\hline Consumo & Consumo & $\begin{array}{l}\text { Consumo mensual de } \\
\text { electricidad en el } \\
\text { hogar. }\end{array}$ & $\begin{array}{l}\text { Cuantitativa } \\
\text { Explicativa }\end{array}$ & Dólares & Positivo \\
\hline Ingreso & Ingreso & $\begin{array}{l}\text { Ingreso mensual en el } \\
\text { hogar. }\end{array}$ & $\begin{array}{l}\text { Cuantitativa } \\
\text { Explicativa }\end{array}$ & Dólares & Positivo \\
\hline \multirow{6}{*}{$\begin{array}{l}\text { Características } \\
\text { Habitantes }\end{array}$} & Integrantes & $\begin{array}{l}\text { Cantidad de } \\
\text { Miembros del hogar. }\end{array}$ & $\begin{array}{l}\text { Cuantitativa } \\
\text { Explicativa }\end{array}$ & Miembros & Positivo \\
\hline & Integrantes $>18$ & $\begin{array}{l}\text { Cantidad de } \\
\text { Miembros mayores de } \\
18 .\end{array}$ & $\begin{array}{l}\text { Cuantitativa } \\
\text { Explicativa }\end{array}$ & Miembros & Positivo \\
\hline & Integrantes $<18$ & $\begin{array}{l}\text { Cantidad de } \\
\text { Miembros menores de } \\
18 .\end{array}$ & $\begin{array}{l}\text { Cuantitativa } \\
\text { Explicativa }\end{array}$ & Miembros & Positivo \\
\hline & Integrantes $>65$ & $\begin{array}{l}\text { Cantidad de } \\
\text { Miembros mayores de } \\
65 .\end{array}$ & $\begin{array}{l}\text { Cuantitativa } \\
\text { Explicativa }\end{array}$ & Miembros & Positivo \\
\hline & Edad & $\begin{array}{l}\text { Edad del jefe del } \\
\text { Hogar. }\end{array}$ & $\begin{array}{l}\text { Cuantitativa } \\
\text { Explicativa }\end{array}$ & Años & Positivo \\
\hline & Sexo & $\begin{array}{l}\text { Sexo del jefe del } \\
\text { Hogar. }\end{array}$ & $\begin{array}{l}\text { Binaria } \\
\text { Cualitativa } \\
\text { Explicativa } \\
\end{array}$ & $1=\mathrm{Si} 0=\mathrm{No}$ & Positivo \\
\hline \multirow{8}{*}{$\begin{array}{l}\text { Características del } \\
\text { Hogar }\end{array}$} & Temporada & $\begin{array}{l}\text { Temporada del año } \\
\text { de mayor consumo. }\end{array}$ & $\begin{array}{l}\text { Binaria } \\
\text { Cualitativa } \\
\text { Explicativa } \\
\end{array}$ & $\begin{array}{l}1=\text { Invierno } \\
\text { (calor) } \\
0=\text { Verano } \\
\end{array}$ & Positivo \\
\hline & $\begin{array}{l}\text { Aire } \\
\text { Acondicionado }\end{array}$ & $\begin{array}{l}\text { Disponibilidad del } \\
\text { uso de aire } \\
\text { acondicionado. }\end{array}$ & $\begin{array}{l}\text { Binaria } \\
\text { Cualitativa } \\
\text { Explicativa }\end{array}$ & $\begin{array}{l}1=\mathrm{Si} \\
0=\mathrm{No}\end{array}$ & Positivo \\
\hline & Ducha Eléctrica & $\begin{array}{l}\text { Disponibilidad del } \\
\text { uso de ducha } \\
\text { eléctrica. }\end{array}$ & $\begin{array}{l}\text { Binaria } \\
\text { Cualitativa } \\
\text { Explicativa } \\
\end{array}$ & $\begin{array}{l}1=\mathrm{Si} \\
0=\mathrm{No}\end{array}$ & Positivo \\
\hline & $\begin{array}{l}\text { Cocina de } \\
\text { Inducción }\end{array}$ & $\begin{array}{l}\text { Disponibilidad del } \\
\text { uso de cocina de } \\
\text { inducción }\end{array}$ & $\begin{array}{l}\text { Binaria } \\
\text { Cualitativa } \\
\text { Explicativa } \\
\end{array}$ & $\begin{array}{l}1=\mathrm{Si} \\
0=\mathrm{No}\end{array}$ & Positivo \\
\hline & Artefactos & Número de aparatos & $\begin{array}{l}\text { Cuantitativa } \\
\text { Explicativa }\end{array}$ & Numero & Positivo \\
\hline & Villa & Vive en villa. & $\begin{array}{l}\text { Binaria } \\
\text { Cualitativa } \\
\text { Explicativa } \\
\end{array}$ & $\begin{array}{l}1=\mathrm{Si} \\
0=\mathrm{No}\end{array}$ & Positivo \\
\hline & Edificio & Vive en edificio & $\begin{array}{l}\text { Binaria } \\
\text { Cualitativa } \\
\text { Explicativa } \\
\end{array}$ & $\begin{array}{l}1=\mathrm{Si} \\
0=\mathrm{No}\end{array}$ & Positivo \\
\hline & Departamento & Vive en departamento & $\begin{array}{l}\text { Binaria } \\
\text { Cualitativa }\end{array}$ & $\begin{array}{l}1=\mathrm{Si} \\
0=\mathrm{No}\end{array}$ & Positivo \\
\hline
\end{tabular}

Esta obra se comparte bajo la licencia Creative Common Atribución-No Comercial 4.0 


\section{Elaborado por autora}

Se utilizaron pruebas de supuestos para corroborar resultados como la de normalidad y homocedasticidad.

\section{Resultados}

La Ciudad de Machala es una de las ciudades más importantes de la Provincia de El Oro, por su comercio formal e informal, consta de siete parroquias, ha presentado cambios en el desarrollo de los habitantes, por el aumento comercial, inversión extranjera, mayores plazas de trabajo, ha repuntado en una mejora de la calidad de vida, sin embargo, los niveles de consumo de servicios básicos, particularmente el de electricidad, están elevándose, para esta investigación fue necesario escoger una de las parroquias con mayor cantidad de hogares como es Jambelí y conocer su comportamiento con características que definen la demanda del servicio básico.

El planteamiento comienza con el análisis de los estadísticos descriptivos de las variables cuantitativas (Consumo, Ingreso, Edad, Integrantes, Integrantes Mas 18, Integrantes Menos 18, Integrantes Mas 65, Artefactos, Temporada, Edificio) para identificar la existencia de datos atípicos que puedan sesgar la información, los mismos que no fueron encontrados en la investigación. Quedando así: Consumo (variable dependiente); Ingreso, Edad, Integrantes, Artefactos, Temporada y Edificio (variables independientes)

La ecuación planteada para el modelo, es la siguiente:

$$
\begin{aligned}
\log (\text { Consumo }) & =\mathrm{C}+\beta \log (\text { Ingreso })+\gamma \log \text { Integrantes }+\delta \log \text { Artefactos } \\
& +\lambda \log \text { Edad }+\theta \text { Temporada }+\varepsilon \text { Edificio }
\end{aligned}
$$

La elección de forma funcional logarítmica en modelo de regresión permite estudiar cuando queremos modelizar cuales variaciones \% (porcentuales) en $\mathrm{X}$ producen variaciones $\%$ constantes en Y, esta reflexión es de gran utilidad para estudios demanda. (Genaro, 2009)

\begin{tabular}{|c|c|c|c|c|c|c|}
\hline Source & SS & df & MS & $\begin{array}{l}\text { Number of obs } \\
=\end{array}$ & 101 & \\
\hline & & & & $\mathrm{F}(6,94)=$ & 24.68 & \\
\hline Model & 25.1372584 & 6 & 4.18954307 & Prob $>F=$ & 0.0000 & \\
\hline \multirow[t]{2}{*}{ Residual } & 15.9550063 & 94 & 0.16973411 & R-squared = & 0.6117 & \\
\hline & & & & R-squared = & 0.5869 & \\
\hline Total & 41.0922647 & 100 & 0.41092264 & Root MSE = & 0.41199 & \\
\hline lconsumo & Coef. & Std. Err. & $\mathrm{t}$ & $\mathrm{P}>\mathrm{t}$ & [95\% Conf. & Interval] \\
\hline lingreso & 0.4753293 & 0.118582 & 4.01 & 0.000 & 0.239880 & 0.710778 \\
\hline lintegrantes & 0.2692113 & 0.130019 & 2.07 & 0.041 & 0.011053 & 0.527368 \\
\hline
\end{tabular}

\section{Tabla. 2. Modelo de Regresión Múltiple Logarítmico}

Esta obra se comparte bajo la licencia Creative Common Atribución-No Comercial 4.0 International (CC BY-NC 4.0) 


\begin{tabular}{lllllll} 
lartefactos & 0.5553977 & 0.096310 & 5.77 & 0.000 & 0.364171 & 0.746624 \\
ledad & 0.4780372 & 0.217552 & 2.20 & 0.030 & 0.046082 & 0.909992 \\
Temporada & 0.5337426 & 0.174996 & 3.05 & 0.003 & 0.186283 & 0.881201 \\
Edificio & 0.2425909 & 0.111384 & 2.18 & 0.032 & 0.021433 & 0.463748 \\
cons & -3.450694 & 0.951064 & -3.63 & 0.000 & -5.33905 & -1.56233 \\
\hline
\end{tabular}

Fuente: Cálculos obtenidos con el software STATA 14 versión de prueba.

Los resultados del modelo, demuestran que el valor de la prueba $\mathrm{F}=24.68$ es mayor a 1 , estableciendo que todas las variables tienen una fuerte relación con la variable explicada, de igual forma la Probabilidad $=0.0000$ afirma que un valor por debajo del nivel de error admisible $5 \%$, considera que las variables independientes son estadísticamente significativas para explicar la dependiente. $\mathrm{Y}$ el R-squared $=0.6117$ refleja que las variables independientes explican un $61 \%$ a la variable dependiente, es decir el modelo se ajusta a los datos.

La nueva ecuación para estimar la demanda del consumo de energía eléctrica, es el siguiente:

LConsumo $=0.4753293($ lingreso $)+0.2692113$ (lintegrantes $)+0.5553977$ (lartefactos $)+$ 0.4780372 (ledad) +0.533742 (Temporada) +0.2425909 (edificio)

Por lo tanto, la incidencia de los coeficientes de las variables ingreso, integrantes del hogar, artefactos, edad, temporada y vivienda, se deduce en:

Ingreso: La elasticidad ingreso de la demanda es positiva e inelástica $(0,47)$, significa que, a mayor nivel de ingreso, ceteris paribus, la demanda aumentará.

Integrantes: La elasticidad de integrantes en la demanda es positiva e inelástica $(0,26)$, significa que, a mayor número de integrantes en el hogar, ceteris paribus, la demanda de energía eléctrica aumentará.

Artefactos: La elasticidad de artefactos en la demanda es positiva e inelástica $(0,55)$. Esto indica que, a mayor número de artefactos eléctricos, ceteris paribus, la demanda de energía eléctrica aumentará.

Edad: La elasticidad de edad en la demanda es positiva e inelástica $(0,47)$. Esto indica, mientras mayor sea la edad del jefe del hogar, ceteris paribus, la demanda de energía eléctrica aumentará.

Temporada: La temporada en la variable es positiva (0,53). Esto indica que, en época de calor los hogares, ceteris paribus, la demanda de energía eléctrica aumentará.

Edificio: Los hogares residentes en edificios presentan una variable positiva $(0,24)$. Esto indica que, mientras los hogares residan en un edificio, ceteris paribus, la demanda de energía eléctrica aumentará.

Tabla. 3. Matriz de Supuestos del Modelo de Regresión Múltiple Logarítmico

\begin{tabular}{ll}
\hline Supuestos del Modelo & \\
\hline Heterocedasticidad & Multicolinealidad \\
Breusch- & VIF $=1.23$
\end{tabular}

Pagan $=0.2712$

Fuente: Cálculos obtenidos con el software STATA 14 versión de prueba.

Esta obra se comparte bajo la licencia Creative Common Atribución-No Comercial 4.0

International (CC BY-NC 4.0)

Revista Trimestral del Instituto Superior Universitario Espíritu Santo 
El supuesto de heterocedasticidad, mediante la prueba Breusch-Pagan obtiene un resultado de 0.2712 , resultando menor que el valor crítico dado en la tabla de $\chi 2$, determinando que los datos residuales están distribuidos homocedásticamente resultando que es significativo para el modelo. Se corroboró el modelo con el factor de inflación de varianza VIF, con un valor de 1.23 , demostrando que todas las variables regresoras presentan valores similares, por lo tanto, no existe problema de Multicolinealidad entre variables, es decir las variables explicativas no están estrechamente relacionadas entre sí.

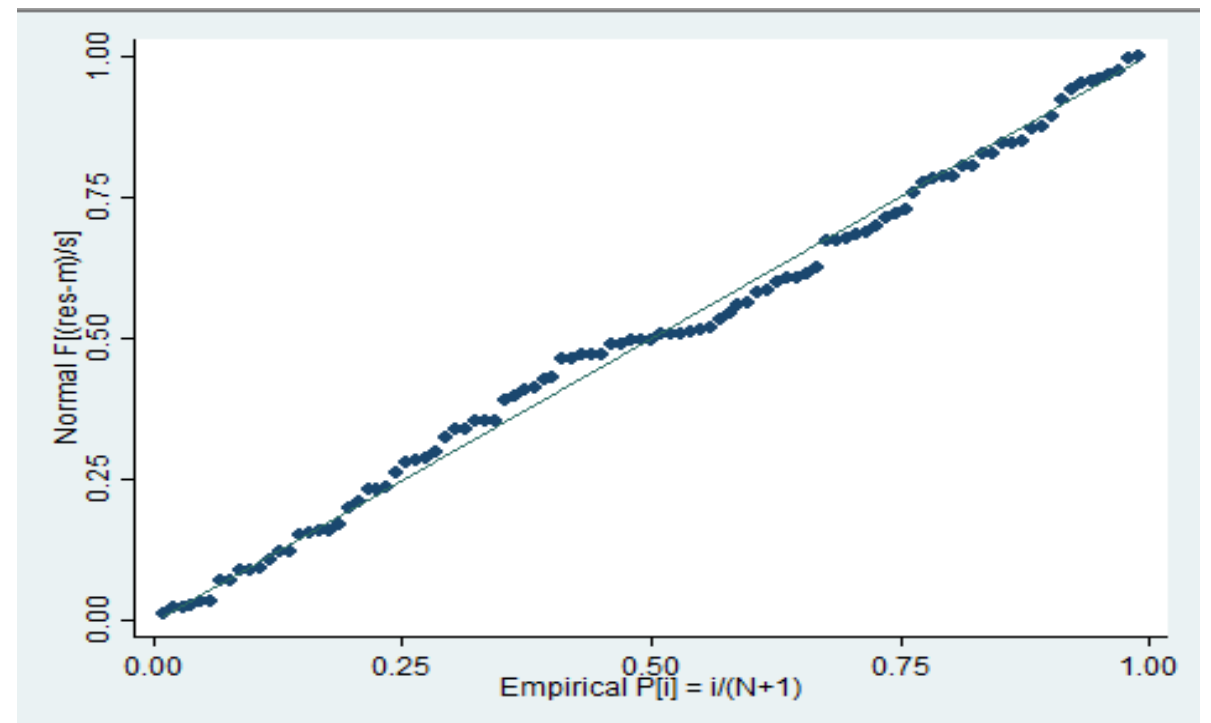

\section{Figura. 1. Probabilidad Normal Regresión Lineal Múltiple}

Fuente: Cálculos obtenidos con el software STATA 14 versión de prueba.

Finalmente se presenta en el Gráfico 1 la probabilidad normal, indicando que los datos residuales siguen un comportamiento de distribución normal. Concluyendo que el modelo es funcional y acertado para las variables propuestas.

\section{Discusión de resultados}

Del análisis de los resultados se establece las principales implicaciones de la demanda del servicio eléctrico en los hogares de la Parroquia Jambelí, tales como:

Los ingresos de los hogares: Desarrollar hábitos de ahorro desde la economía familiar, en el destino de gastos necesarios, provisionales y de lujo. Se debe orientar a la comunidad de estudio en la creación de estilos de vida sostenibles y proporcionen una cultura doméstica de consumo responsable del servicio básico.

Edad: La edad es un factor que incide en el patrón de consumo, se debe considerar horarios de uso de los principales artefactos eléctricos. Establecer subsidios de energía eléctrica por parte de la autoridad de gobierno en los hogares con mayor número de integrantes e ingresos

Esta obra se comparte bajo la licencia Creative Common Atribución-No Comercial 4.0 International (CC BY-NC 4.0) 
que no superen el salario básico unificado, es una forma de apoyo social y disciplina de demanda.

Integrantes de los hogares: Ofrecer capacitaciones de finanzas en el hogar sobre el uso del servicio eléctrico desde la actuación de cada integrante del hogar para evitar perdida de la capacidad de ahorro. Promover campañas de reserva y responsabilidad social en la familia y comunidad.

Artefactos eléctricos: Aplicar normas de uso familiar de los aparatos eléctricos, es decir, mientras mayor sea la calidad de vida, aumentará el consumo de electricidad reflejado en la demanda del hogar. Concientizar a los hogares de la localidad sobre el comportamiento energético en tiempos de crisis y bonanza económica.

Temporada: Las altas temperaturas típicas de la ciudad de Machala generan aumento del kilowatt-hora en los hogares, puede provocar fallos en los suministros de electricidad, ante estas estaciones climáticas se debe proponer mecanismos de apoyo por parte de la autoridad gubernamental para evitar que otros hogares y locales comerciales se queden sin dicho servicio. Incentivar en la elección de aparatos de climatización más adecuados y de etiqueta ecológica.

Vivienda (Edificio): Usar la energía eléctrica sólo cuando sea necesario, por lo tanto, una serie de políticas, instrucciones e incentivos ayudarán a usar los recursos de la mejor manera. Incentivar el desarrollo de programas de ahorro de energía, como de eficiencia energética a los abonados del sector.

Existen varias aplicaciones fijas de ahorro eléctrico en el hogar, que conducen a una lista de medidas de conservación con planificación estratégica, inteligente y creativa.

\section{Conclusiones}

La aplicación del instrumento de investigación test/encuesta, permite obtener datos importantes para la determinación de la demanda del servicio eléctrico de los hogares de la Parroquia Jambelí, siendo necesario considerar variables que influyen en el consumo.

Se corrobora la mención de Keynes sobre el consumo a causa de la demanda determinada por una de las variantes principales como es el ingreso. El procesamiento de datos mediante modelación econométrica permitió verificar la calidad del modelo, deduciendo ser funcional y adecuado. Del análisis de la ecuación demanda de los hogares se deduce, que a medida que aumentan los ingresos del hogar, número de integrantes, compra de artefactos eléctricos, edad de los integrantes de las familias, a medida que llega la temporada de calor y mientras vivan en edificios, la demanda de servicio energético será mayor, causando efectos en la capacidad de ahorro de los hogares por el aumento de pago de las planillas de luz.

Los resultados anteriores demuestran la obligatoriedad de control por parte de los

Esta obra se comparte bajo la licencia Creative Common Atribución-No Comercial 4.0 
Gobiernos Descentralizados, mejorar las políticas públicas con planteamientos de otorgar a los hogares planes y técnicas para el ahorro de energía eléctrica, cuyos beneficios se reflejarán en un menor consumo y menor facturación. Sin embargo, es prioridad la aplicación del instrumento en las demás parroquias de la ciudad de Machala y establecer comparativos de consumo y ahorro de electricidad.

\section{Referencias Bibliográficas}

Agencia de Regulación y Control de Electricidad (ARCONEL) (2002). Codificacion del Reglamento de Tarifas Eléctricas. Decreto Ejecutivo No. 2713. R.O. No. 598. Obtenido de https://www.regulacionelectrica.gob.ec/wpcontent/uploads/downloads/2015/11/ReglamentosVigentes.html

Agencia de Regulación y Control de Electricidad (ARCONEL) (2011). Estadística del Sector Eléctrico Ecuatoriano. Directorio del Consejo Nacional de Electricidad. Obtenido den https://www.regulacionelectrica.gob.ec/wpcontent/uploads/downloads/2015/11/Folleto-Resumen-Estad\%C3\%ADsticas-2011.pdf

Álvarez, M. (2016). La liberalización en la industria eléctrica española. El reto de lograr precios competitivos para los hogares. Gestión y Política Pública . (Vol.XXV) ํ2, 551-589.

Caporaso, J., \& Levine, D. (1992). Politics and economics. Economy. Cambirdge: Cambridge University Press. 100-120.

Carosio, A. (2008). El género del consumo en la sociedad de consumo. Revista La Ventana. Vol. III. N²7. 130-169.

Castells, X. E. (2011). Energía, Agua, Medioambiente, Territorialidad y Sostenibilida. Madrid: DiazSantos. Obtenido de https://www.editdiazdesantos.com/wwwdat/pdf/9788499690094.pdf

Cayuela, L. (2010). Modelos lineales: Regresión, ANOVA y ANCOVA. Universidad de Granada-Junta de Andalucía. Obtenido de http://recursos.salonesvirtuales.com/assets/bloques/2-Modelos-lineales_luis_cayuela.pdf

Del Canto, E., \& Silva, A. (2013). Metodología Cuantitativa: Abordaje desde la complementariedad en Ciencias Sociales. Revista de Ciencias Sociales. (Vol.III) $\mathrm{N}^{\circ} 141$. 25-34.

García, A. (2010). Oferta y demanda y el ciclo económico: una interpretación de la situación económica actual. Revista Estudios de Economía Aplicada. (Vol. 28) N⒊671685.

Genaro, S. (2009). El Modelo de regresion lineal Multiple. Departamento de Economía. Econometría 1 curso 2009-2010. 1-47. Obtenido de http://www.sucarrat.net/teaching/econometria1/2010/tema03.pdf

Gujarati, D., \& Porter, D. (2010). Econometría. México: McGraw Hill. 5ta. Edicion. $1-921$.

Hernandez, R., Fernandes, C., \& Baptista, M. (2010). Metodologia de la Investigacion. Mexico: Marcela I. Rocha Martínez. México: McGRAW-HILL 5ta. Edición. Obtenido de 
https://www.esup.edu.pe/descargas/dep_investigacion/Metodologia\%20de\%201a\%20invest igaci\%C3\%B3n\%205ta\%20Edici\%C3\%B3n.pdf

Mejía, J. (2016). Las concepciones de bienestar y el consumo. Un análisis

bibliográfico desde la economía civil y las ciencias sociales. Revista Otra Economia. (Vol. 10) $\mathrm{N}^{\circ} 19.241-252$.

Monje, C. (2011). Metodología de la Investigación Cualitativa y Cuantitativa. Universidad SurColombiana. Facultad de Ciencias Solciales y Humanas. Programa de Comunicación Social y Periodismo. Neiva. 1-216. Obtenido de https://www.uv.mx/rmipe/files/2017/02/Guia-didactica-metodologia-de-lainvestigacion.pdf

Morales, D., \& Alvarado, E. (2014). Análisis del consumo de energía eléctricca domiciliaria en Tampico Tamaulipas. Revista Ciencia UAT. (Vol.8) N².62-67.

OLADE (2019). Logros Instirucionales en beneficio a países miembros. Organización Latinoamérica de Energía (OLADE). Revista de Energía de Latinoamérica y el Caribe. (Vol.II) $\mathrm{N}^{\circ}$ 2. 1-110.

Pavón, C., \& Barzola, J. (2015). Estimación de la demanda energética mensual mediante encuesta aplicada en la Provincia de Santa Elena. Revista YACHANA. (Vol.4) $\mathrm{N}^{\circ} 2.22-29$. 